\title{
Superconducting Radio Frequency Resonators for Quantum Computing: A short Review
}

\section{Pashupati Dhakal}

Journal of Nepal Physical Society

Volume 7, No 3, 2021

(Special Issue: ANPA Conference, 2021)

ISSN: 2392-473X (Print), 2738-9537 (Online)

\section{Editors:}

Dr. Nabin Malakar (Editor in chief)

Worcester State University

Dr. Pashupati Dhakal

Thomas Jefferson National Accelerator Facility, USA Dr. Arjun Dahal

University of South Alabama, USA

Dr. Chiranjivi Lamsal

SUNY Platsburgh, USA

Dr. Dilli Raj Paudyal

University of Regina, Canada

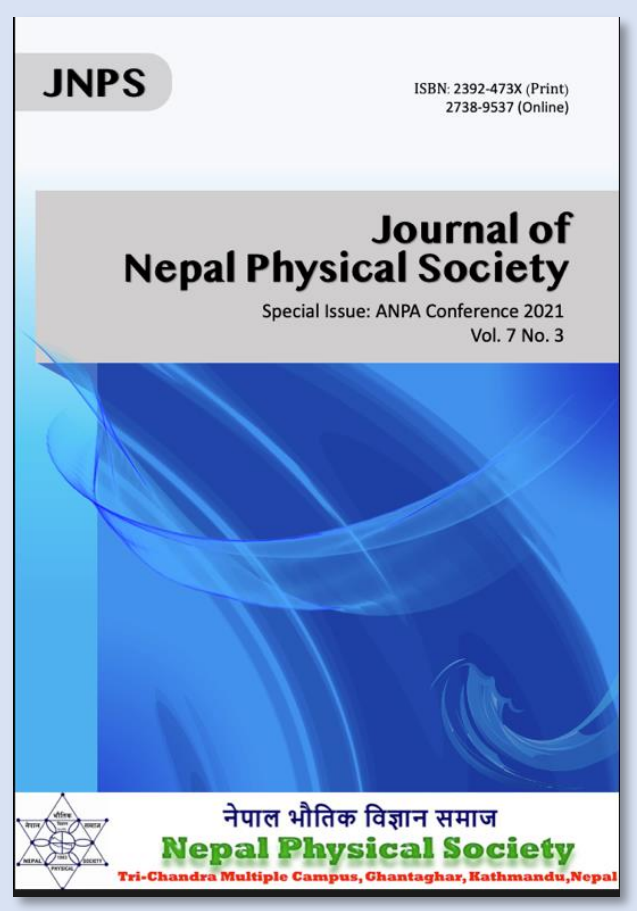

Managing Editor:

Dr. Binod Adhikari

St. Xavier's College, Kathmandu, Nepal

JNPS, 7 (3), 1-5 (2021)

DOI: http://doi.org/10.3126/jnphyssoc.v7i3.42179

Published by: Nepal Physical Society

P.O. Box: 2934

Tri-Chandra Campus

Kathmandu, Nepal

Email: npseditor@gmail.com 


\title{
Superconducting Radio Frequency Resonators for Quantum Computing: A Short Review
}

\author{
Pashupati Dhakal ${ }^{\text {a) }}$ \\ Thomas Jefferson National Accelerator Facility, Newport News VA 23606, USA

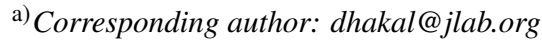

\begin{abstract}
Use of the superconducting resonators deliver superior performance due to their high quality factor and therefore longer decay time of the stored energy. An emerging application of these superconducting resonators is for quantum computing and quantum information science enabling discoveries that explore and deepen our understanding of matter that might otherwise be impossible to explore with classical computing and technology. The quantum processing architecture uses resonators and interconnecting circuits operating in the microwave regime with superconducting strip-line technology and low noise electronic devices for switching and communication. The performance of these devices can be enhanced by embedding them in three dimensional resonators to prolong the coherence time, which improves the utility of the device by reducing error rates and allowing more manipulations (calculations) before the quantum state decays. Here, we present a short review of current microwave technology used in quantum computing and progress towards enhancing the qubit coherence time.
\end{abstract}

Received: 14 August 2021; Revised: 30 October 2021; Accepted: 15 November 2021

Keywords: Microwave Resonators, Superconductivity, Quantum Computers, Qubits

\section{INTRODUCTION}

Classical computing units or "bits" rely on two binary states " 0 " and " 1 ". This is sufficient for many everyday problems and forms the basis of our modern information society. However, it can become a limitation for certain complex problems of exponential scale. The limitation can be overcome with a quantum bit, aka "qubit" which can contain a lot more information by employing the superposition of two quantum mechanical states $\mid 0>$ and $\mid 1>$ in Hilbert space (a two-dimensional complex vector space), as shown in Fig. 1. Study of quantum superposition uncovers the intriguing quantum phenomena of entanglements [1,2] and coherence [3]; vital for applications in quantum processing and computing, quantum metrology [4], and cryptography [5] to name a few. Recently, a processor with programmable qubits was demonstrated to create quantum states on 53 qubits sampling over few minutes, whereas an equal fidelity classical sampling using state-of-art classical computing would have taken several years [6]. While quantum computers won't replace the existing computers in our daily life that can fit in the palm of the hand, they will offer the capability to perform calculations which may otherwise be impossible using current computing technology.

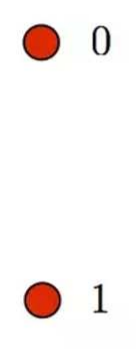

Classical bit

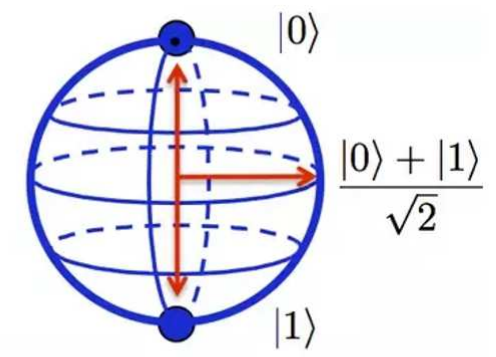

Qubit

FIGURE 1. Bloch sphere with a vector $\mid \xi>$ in Hilbert space represents the quantum state or qubit state. The two states $|0\rangle$ and $I 1>$ are ground and excited state.

In quantum computing, one must be able to create the superposition of two states and maintain their quantum coherence, defined as the length of time for which the qubit maintains reliably the given superposition of quantum states with enough fidelity to allow a series of quantum manipulations to be performed [7]. One of the requirements for optimal performance of quantum com- 
puters is perfect isolation of qubit from the outside world but in such a way that an external user has the capability to control, manipulate and read out. With respect to the Hilbert space as shown in Fig. 1, there are two characteristic times that contribute to the coherence loss; longitudinal relaxation $T_{1}$ corresponding to the energy decay rate (absorption or emission) of a two level system and transverse relaxation $T_{2}$ corresponding to the time for which the qubit remains on phase coherent state [8]. In most systems $T_{1}>T_{2}$, meaning the phase coherence is more important for the quantum system following the $\mathrm{Di}$ Vincenzo criteria [9]. Over the last two decades, the qubit life time reached milliseconds and number of qubits in a chips reached more than 50 qubits as shown in Fig. 2. To

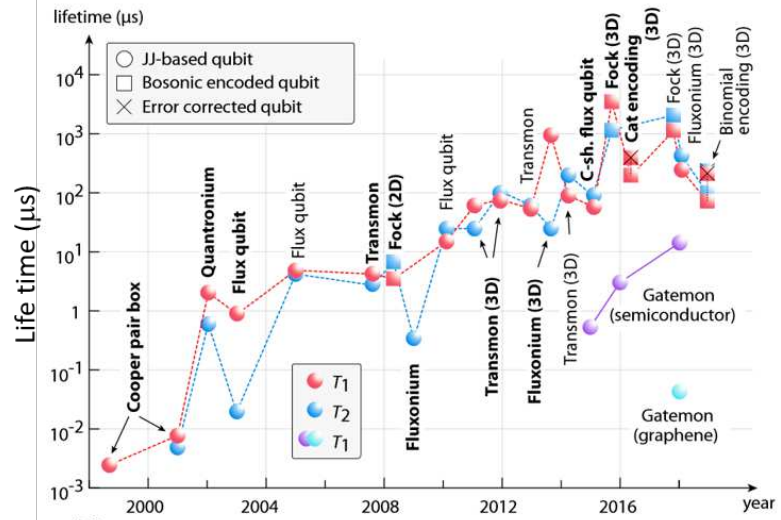

(a)

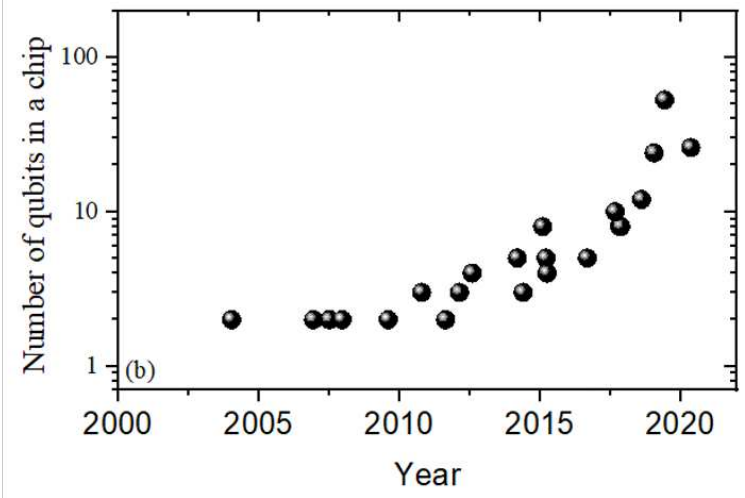

FIGURE 2. Development of superconducting qubit. (a) Qubit coherence time [10] and (b) the number of qubit on chip [11]

physically implement a quantum computer, several candidates are being pursued to realize qubits. These include: superconducting qubits, trapped ion, quantum dots, spins states of trapped electrons, nitrogen vacancy centers in diamonds, quantum wires, nuclear spins, etc [12]. One implementation of quantum processing architecture uses resonators and interconnecting circuits operating in the microwave regime employing superconducting strip-line technology and low noise electronic devices for switching and communication. Quantum devices rely on the nonlinearity of one or more Josephson junctions (JJ) in the circuit which are dissipationless and stable to avoid decoherence. The Josephson junction creates a nonzero periodic current due to tunneling Cooper pairs across the superconductor-insulator-superconductor junction acting as a macroscopic quantum variable. The Josephson junction can be used to produce an anharmonic LC oscillator where discretized energy levels are unequally spaced and by isolating two levels, one can realize a two level systems which can store one qubit of information as shown in Fig. 3. This qubit can be coupled with microwave cavities that coherently transmit quantum information [13]. The coherence of superconducting qubits depends on two distinct aspects; the stability of the Josephson junction itself and the degree of interaction with the environment. In cavity quantum electrodynamics, a superconducting
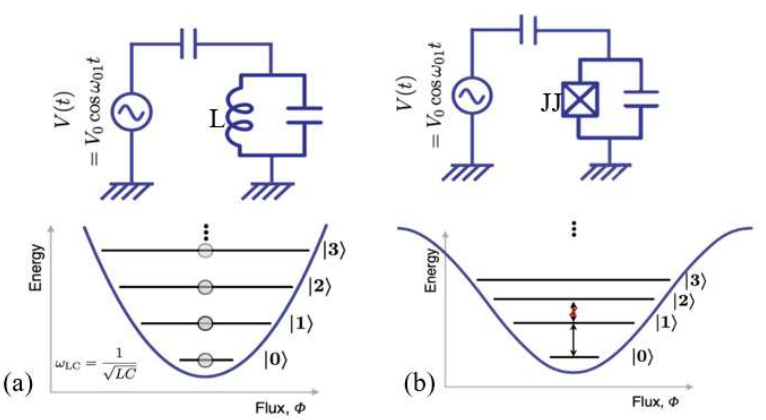

FIGURE 3. (a) LC oscillator circuit behaving a harmonic oscillator with equally spaced energy levels. (b) Equivalent LC oscillator with inductor replaced by Josephson junction behaving an anharmonic oscillator.

charge qubit consisting of a JJ shunted by a large capacitor is called "transmon" and is coupled to a microwave cavity. By using superconducting microwave cavities, it is possible to increase coherence time of qubit. Planar on-chip transmon qubits have a coherence time $\sim 30-40$ $\mu \mathrm{s}$, however a coherence time over $1 \mathrm{~ms}$ was achieved using a three dimensional superconducting cavity [10]. The carefully engineered interactions between the physical qubits and the cavity offer a wonderful architecture for quantum information processing [14, 15]. The high quality factor superconducting microwave cavities essentially act as a narrow-band filter allowing isolation from the unwanted interactions with the outside world. The microwave cavities provide the resources to mediate entanglement between the physical qubits, measure qubit states and store the quantum information [16]. It has been proposed that $3 \mathrm{D}$ cavities interacting with transmon can create a superposition of two arbitrary states in two cavities, a quantum adder phenomenon for quantum information processing $[17,18]$. Furthermore, the coupled system of superconducting qubit and 3D cavities offer 
excellent capabilities for creating quantum cavity states through the nonlinearly of intermediary qubits $[19,20$, $21,22,23]$. In this manuscript, we briefly review the current status of superconducting resonators and research towards the development of $3 \mathrm{D}$ resonators in order to increase the qubit coherence time.

\section{D SUPERCONDUCTING QUBIT SYSTEM}

The superconducting qubit system is lithographically fabricated to make an "artificial atom" behaving like the two level system (TLS) typically used in three fundamental qubit modalities: charge, flux and phase as well as hybridization to transmon, fluxonium, quantronium. The qubit, superconducting circuits, interconnects and microwave resonators provides the entanglement between the qubits and a means to measure the qubit state as well as store the quantum information [24]. Figure 4 shows the image of the of IBM's five qubit processor along with the qubits, microwave resonators fabricated with superconducting materials on substrate. Despite the advantages of the controlled fabrication of the superconducting circuits in 2D geometries, the qubit system suffers a low coherence time. There are several sources of loss mechanism responsible to the performance, for example the dielectric loss arising from the interfaces between the superconducting materials and the substrate, excess quasi-particles in superconducting material, oxides and contaminants for two level system, surface spins and charge noise that cause the dissipation and dephasing [25]. The Josephson junction in superconducting qubits are mainly fabricated from thin film of aluminum $\left(T_{c} \sim\right.$ $1.2 \mathrm{~K}$ ) making $\mathrm{Al} / \mathrm{AlO} \mathrm{O}_{x} / \mathrm{Al}$ junction due to the reason that the Al can be easily evaporated on the substrate and $A l O_{x}$ can be grown in controlled thickness and have less pinholes which reduce the leakage current in junction. As shown in Fig. 4, the niobium capacitor pads are deposited to tune the frequency of qubit and the combination of the Josephson junction and capacitor acts as superconducting qubit coupled with microwave resonators. Superconducting qubits can store a significant fraction of the electric field in the interface of the superconductor and substrate enhancing the loss as a result of the absorption of charged ions or dipoles and reemission as phonons, which decreases the coherence time of qubit. Another source of the loss mechanism is due to the two-level systems in the substrate and the non-equilibrium quasi-particles. The TLS related loss increase with the decreasing the microwave power at low temperatures [26]. The qubit's electric field couples with the TLS and resulting in a lower coherence time. Several current research works focused on dependence of loss mechanism to the geometry, fabrication parameters. The much lower current state- of-the-art qubit life time than those predicted by known loss mechanism suggest that the surfaces and interfaces play the significant role in limiting the coherence time in superconducting qubits $[25,26,27]$. Understanding

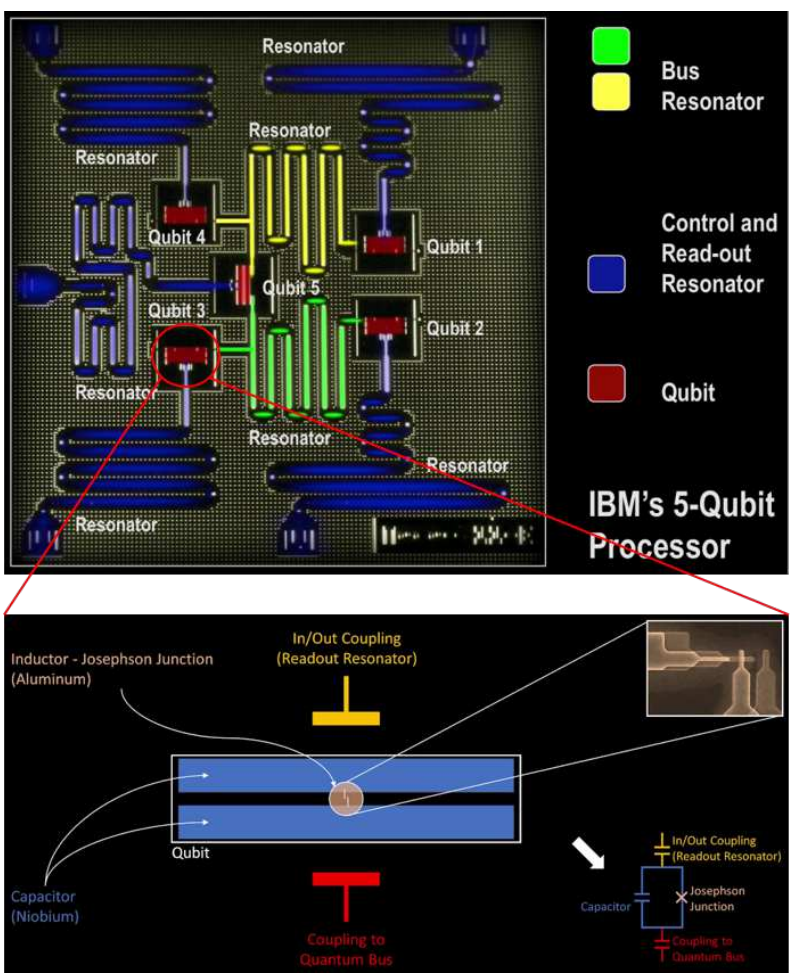

FIGURE 4. An image of IBM's five qubit processor with its main elements highlighted, including the five qubits and their control, read out and coupling resonators (top). A schematic closeup of IBM's superconducting qubit, including a zoomed-in view of its Josephson junction (bottom). Taken from Ref. [28]

the mechanisms of loss in superconducting qubits is still an active and important area of research. It has become clear that high quality superconducting films and careful processing of these films is required for reduced loss and consequently longer coherence time. Currently, high quality $\mathrm{Nb}$ and $\mathrm{NbTiN}$ thin films are being perused to fabricate the superconducting qubit architectures. Recently, a significant improvement over the state of the art in $2 \mathrm{D}$ transmon qubits was achieved by using tantalum as the superconductor in the capacitor and microwave resonators, replacing the more commonly used niobium [29].

\section{D SUPERCONDUCTING QUBIT SYSTEM}

Current 3D microwave cavities such as those shown in Fig. 5, are made from high purity aluminum with super- 
conducting transition temperature $\sim 1.2 \mathrm{~K}$. Aluminum is a low temperature superconductor and a material easy to machine to a complex shape, has led to its widespread use. As mentioned, 2D resonators have low coherence times mostly due to dielectric losses at the interfaces of the superconductors and substrates [10], whereas the 3D geometry may provide longer lifetimes due to the increased stored energy and very low wall losses since the most of the electric field is stored in the vacuum. In addition, the higher superconducting energy gap of $\mathrm{Nb}$ may be beneficial in operating the cavity at higher frequency providing a more compact structure. In an attempt to use $\mathrm{Nb}$ cavities for quantum applications, Fermi Lab recently reported the measurement of the quality factor of these accelerating resonators down to $\mathrm{T} \sim 20 \mathrm{mK}$ and demonstrated a high quality factor $\sim 10^{10}$ corresponding to photon lifetime $\sim$ 2 seconds [30]. They showed that the saturation of quality factor may arise from the TLS in the niobium oxide, however this might be overcome with thermal treatments. SRF cavities made from superconducting niobium rou-
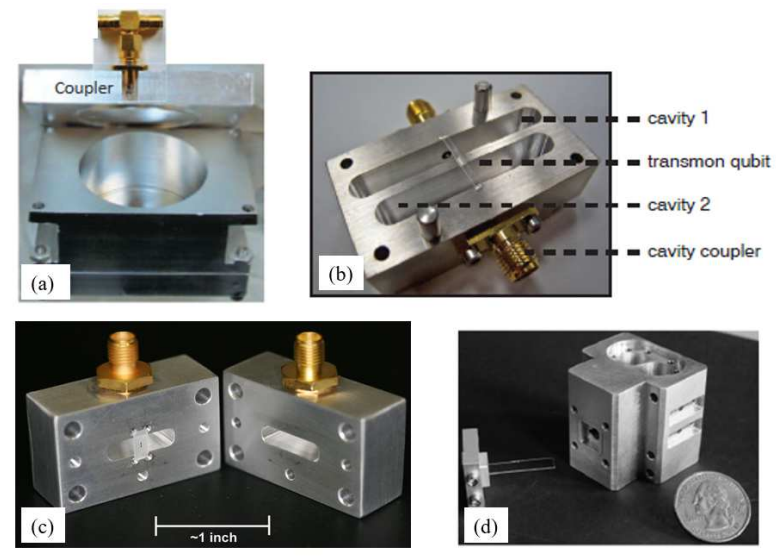

FIGURE 5. Three dimensional resonator configurations. (a) Schematic of cylindrical resonator in shunt configurations [27], (b) Two cavity resonator with a sapphire chip with a lithographically patterned transmon qubit [21], (c) 3D rectangular Al cavity showing the interior volume of the waveguide enclosure housing a sapphire chip and transmon qubit [14], (d) 3D version of a quarter wave resonator with a Y-shaped transmon type superconducting qubit [31]

tinely achieve $Q_{0} \sim 10^{10}-10^{11}$ corresponding to several seconds of photon lifetime, higher than the reported 3D microwave cavities made from $\mathrm{Al}$ [32]. However, the SRF cavities are optimized forparticle accelerators and not well optimized for the quantum computing applications. Figure 6 shows the intrinsic quality factor of 1.5 $\mathrm{GHz}$ accelerating SRF cavity as a function of temperature. The quality factor of these SRF cavities in quantum regime $(\sim 20 \mathrm{mK})$ has not been fully understood. There are several 3D niobium cavities that are being explored to optimize for qubit integration [33].

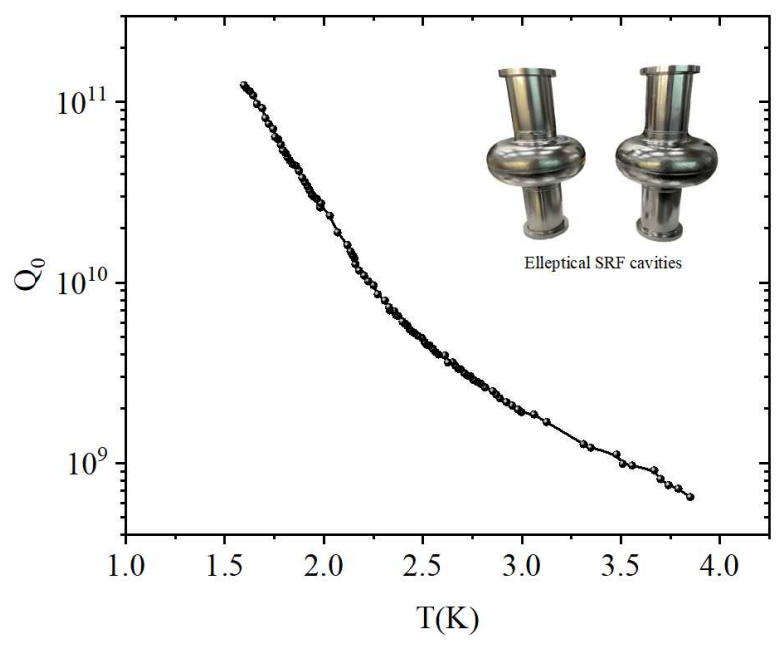

FIGURE 6. Temperature dependence of intrinsic qualify factor of $1.5 \mathrm{GHz}$ SRF elliptical cavity in the temperature range 4.0$1.6 \mathrm{~K}$. The quality factor of $>10^{11}$ corresponds to the photon life time of few seconds.

\section{SUMMARY}

Superconducting radio frequency technology is being used not only in discovery science programs in powerful accelerators and basic research but also for several applications that benefit society more directly. The advantage of superconducting resonators over those made of normal conducting metal is their ability to store electromagnetic energy with much lower dissipation [34]. Building quantum computers with superconducting microwave resonators and circuits with Josephson junction as qubit to create an anharmonic oscillator is rapidly progressing towards the millisecond qubit coherence time. The research and development in resonator design improved the performance. The environmental radiation is found to limit the performance of superconducting qubit [35]. At present, the number of qubits that can be manipulated has already surpassed 50 qubits. Some of the challenges still remaining are to increase the coherence time, quantum gate operations, and readout fidelity with superconducting qubits, quantum error correction needed to realize in large scale quantum computers [36].In addition to their application in accelerator and quantum computers, microwave cavities are extensively used in axion dark matter detection.

\section{ACKNOWLEDGMENTS}

We would like to acknowledge Dr. Gianlugi Ciovati and Dr. Robert Rimmer for their support. This manuscript is authored by Jefferson Science Associates, LLC under 


\section{U.S. DOE Contract No. DE-AC05-06OR23177.}

\section{EDITOR'S NOTE}

This manuscript was submitted to the Association of Nepali Physicists in America (ANPA) Conference 2021 for publication in the special issue of Journal of Nepal Physical Society.

\section{REFERENCES}

1. R. Horodecki, P. Horodecki, M. Horodecki, and K. Horodecki, "Quantum entanglement," Rev. Mod. Phys. 81, 865-942 (2009).

2. Y. Y. Gao, B. J. Lester, K. S. chou, L. Frunzio, M. H. Devoret, L. Jiang, S. M. Grivin, and R. J. Schoelkopf, "Entanglement of bosonic modes through an engineered exchange interaction," Nature 566, 509-512 (2019)

3. T. Baumgratz, M. Cramer, and M. B. Plenio, "Quantifying coherence,” Phys. Rev. Lett. 113, 140401 (2014).

4. V. Giovannetti, S. Lloyd, and L. Maccone, "Advances in quantum metrology," Nature Photonics 5, 222-229 (2011).

5. N. Gisin, G. Ribordy, W. Tittel, and H. Zbinden, "Quantum cryptography,” Rev. Mod. Phys. 74, 145-195 (2002).

6. E. Arute et al.,, "Quantum supremacy using a programmable superconducting processor," Nature 574, 505-510 (2019).

7. R. Vijay, D. H. Slichter, and I. Siddiqi, "Observation of quantum jumps in a superconducting artificial atom," Phys. Rev. Lett. 106 110502 (2011).

8. J. M. Martinis, "Superconducting phase qubits," Quantum Inf. Process 8, 81-103 (2009).

9. D. P. DiVincenzo, "The physical implementation of quantum computation," Fortschritte der Physik 48, 771-783 (2000).

10. M. Kjaergaard, M. E. Schwartz, J. Braumüller, P. Krantz, J. I.-J. Wang, S. Gustavsson, and W. D. Oliver, "Superconducting qubits: Current state of play," Annual Review of Condensed Matter Physics 11, 369-395 (2020).

11. S. Shiro and H. Gotoh, "Activities toward new principle computers," https://www.ntt-review.jp/archive/ntttechnical.php?contents= ntr202105fa1.html, accessed: 2021-06-20.

12. T. D. Ladd, F. Jelezko, R. Laflamme, Y. Nakamura, C. Monroe, and J. L. O'Brien, "Quantum computers," Nature 464, 45-53 (2010).

13. I. Chiorescu, Y. Nakamura, C. J. P. M. Harmans, and J. E. Mooij, "Coherent quantum dynamics of a superconducting flux qubit," Science 21, 1869-1871 (2003).

14. A. Blais, R.-S. Huang, A. Wallraff, S. M. Girvin, and R. J. Schoelkopf, "Cavity quantum electrodynamics for superconducting electrical circuits: An architecture for quantum computation," Phys. Rev. A 69, 062320 (2004).

15. A. Wallraff, D. I. Schuster, A. Blais, L. Frunzio, R.-S. Huang, J. Majer, S. Kumar, S. M. Girvin, and R. J. Schoelkopf, "Strong coupling of a single photon to a superconducting qubit using circuit quantum electrodynamics," Nature 431, 162-167 (2004).

16. S. M. Girvin and R. J. Schoelkopf, "Wiring up quantum systems," Nature 451, 664-669 (2008).

17. M. Oszmaniec, A. Grudka, M. Horodecki, and A. Wójcik, "Creating a superposition of unknown quantum states," Phys. Rev. Lett. 116, 110403 (2016)

18. T. Liu, Y. Zhang, B.-Q. Guo, C.-S. Yu, and W.-N. Zhang, "Creation of superposition of arbitrary states encoded in two high-q cavities," Opt. Express 27, 27168-27182 (2019).

19. W. D. Oliver and P. B. Welander, "Materials in superconducting quantum bits," MRS Bulletin 38, 816-825 (2013).
20. J. Joo, C.-W. Lee, S. Kono, and J. Kim, "Logical measurementbased quantum computation in circuit-qed," Scientific Reports 9, 16592 (2019)

21. B. Vlastakis, G. Kirchmair, Z. Leghtas, S. E. Nigg, L. Frunzio, S. M. Girvin, M. Mirrahimi, M. H. Devoret, and R. J. Schoelkopf, "Deterministically encoding quantum information using 100-photon schrödinger cat states," Science 342, 607-610 (2013).

22. D. I. Schuster, A. A. Houck, J. A. Schreier, A. Wallraff, J. M. Gambetta, A. Blais, L. Frunzio, J. Majer, B. Johnson, M. H. Devoret, S. M. Girvin, and R. J. Schoelkopf, "Resolving photon number states in a superconducting circuit," Nature 445, 515-518 (2007).

23. S. E. Nigg, H. Paik, B. Vlastakis, G. Kirchmair, S. Shankar, L. Frunzio, M. H. Devoret, R. J. Schoelkopf, and S. M. Girvin, "Black-box superconducting circuit quantization," Phys. Rev. Lett. 108, 240502 (2012).

24. J. Koch, T. M. Yu, J. Gambetta, A. A. Houck, D. I. Schuster, J. Majer, A. Blais, M. H. Devoret, S. M. Girvin, and R. J. Schoelkopf, "Charge-insensitive qubit design derived from the cooper pair box," Phys. Rev. A 76, 042319 (2007).

25. N. P. de Leon, K. M. Itoh, D. Kim, K. K. Mehta, T. E Northup, H. Paik, B. S. Palmer, N. Samarth, S. Sangtawesin, and D. W. Steuerman, "Materials challenges and opportunities for quantum computing hardware," Science 372 (2021), 10.1126/science.abb2823.

26. J. M. Martinis, K. B. Cooper, R. McDermott, M. Steffen, M. Ansmann, K. D. Osborn, K. Cicak, S. Oh, D. P. Pappas, R. W. Simmonds, and C. C. Yu, "Decoherence in josephson qubits from dielectric loss," Phys. Rev. Lett. 95, 210503 (2005).

27. O. Dial, D. T. McClure, S. Poletto, G. A. Keefe, M. B. Rothwell, J. M. Gambetta, D. W. Abraham, J. M. Chow, and M. Steffen, "Bulk and surface loss in superconducting transmon qubits," Superconductor Science and Technology 29, 044001 (2016).

28. "IBM Research," https://www.ibm.com/quantum-computing/quan tum-computing-at-ibm.

29. A. P. M. Place, L. V. H. Rodgers, P. Mundada, B. M. Smitham, M. Fitzpatrick, Z. Leng, A. Premkumar, J. Bryon, A. Vrajitoare, S. Sussman, G. Cheng, T. Madhavan, H. K. Babla, X. H. Le, Y. Gang, B. Jäck, A. Gyenis, N. Yao, R. J. Cava, N. P. de Leon, and A. A. Houck, "New material platform for superconducting transmon qubits with coherence times exceeding 0.3 milliseconds," Nature Communications 12, 1779 (2021)

30. A. Romanenko, R. Pilipenko, S. Zorzetti, D. Frolov, M. Awida, S. Belomestnykh, S. Posen, and A. Grassellino, "Threedimensional superconducting resonators at $20 \mathrm{mk}$ with photon lifetimes up to $\tau=2 \mathrm{~s}$," Phys. Rev. Applied 13, 034032 (2020).

31. C. Wang, Y. Y. Gao, P. Reinhold, R. W. Heeres, N. Ofek, K. Chou, C. Axline, M. Reagor, J. Blumoff, K. M. Sliwa, L. Frunzio, S. M. Girvin, L. Jiang, M. Mirrahimi, M. H. Devoret, and R. J. Schoelkopf, "A schrödinger cat living in two boxes," Science $\mathbf{3 5 2}$ 1087-1091 (2016).

32. P. Dhakal, "Nitrogen doping and infusion in srf cavities: A review," Physics Open 5, 100034 (2020).

33. S. V. Kutsaev, K. Taletski, R. Agustsson, P. Carriere, A. N. Cleland, Z. A. Conway, E. Dumur, A. Moro, and A. Y. Smirnov, "Niobium quarter wave resonator with the optimized shape for quantum information systems," EPJ Quantum Technol. 7, 7 (2020).

34. H. Padamsee, "50 years of success for srf accelerators-a review," Supercond. Sci. Technol. 30, 053003 (2017).

35. A. P. Vepsäläinen, A. H. Karamlou, J. L. Orrell, A. S. Dogra, B. Loer, F. Vasconcelos, D. K. Kim, A. J. Melville, B. M. Niedzielski, J. L. Yoder, S. Gustavsson, J. A. Formaggio, B. A. VanDevender, and W. D. Oliver, "Impact of ionizing radiation on superconducting qubit coherence," Nature 584, 551-556 (2020).

36. R. Bradley, J. Clarke, D. Kinion, L. J. Rosenberg, K. van Bibber, S. Matsuki, M. Mück, and P. Sikivie, "Microwave cavity searches for dark-matter axions," Rev. Mod. Phys. 75, 777-817 (2003). 